\title{
Visión de la gestión del cuidado del profesional de enfermería en el primer nivel de atención de las microredes de salud de Tacna
}

Care Management Vision of Nursing Proffesionals in the First Care Level in Health System MicroNetworks Located in Tacna

Visão da gestão do cuidado profissional de enfermagem no primeiro nível de atenção

Karimen Jetzabel Mutter Cuellar ${ }^{1 a}$

D https://orcid.org/0000-0003-3651-7514

Elena Cachicatari Vargas ${ }^{1 a}$

(iD) https://orcid.org/0000-0002-9843-432X

\section{Resumen}

Objetivo: Analizar el significado de la visión de la gestión del cuidado de los profesionales de enfermería que trabajan en el primer nivel de atención de las micro redes de salud de Tacna. El escenario tomó lugar en la provincia y ciudad de Tacna. Los participantes fueron 07 profesionales de enfermería con cargo o experiencia en Jefatura de las micro redes y establecimientos de salud. Material y métodos: Estudio cualitativo de teoría fundamentada. La técnica fue la entrevista a profundidad y como instrumento una guía de entrevista semiestructurada. La muestra se obtuvo del muestreo teórico por saturación de datos. Resultados: En el análisis de la información se utilizó el método de teoría fundamentada mediante el proceso de codificación abierta, axial y selectiva en el que emergieron cinco categorías, 13 subcategorías y 28 unidades de análisis. La primera categoría estuvo relacionada a los conceptos disciplinarios; la segunda, al juicio profesional de la gestión del cuidado; como tercera categoría, se consideró la competencia del profesional de enfermería para la gestión del cuidado; la cuarta categoría estuvo en relación con la comunidad; finalmente, la quinta categoría fue sobre liderazgo. De ellas emergió la teoría que representa la visión de la gestión del cuidado. Conclusiónes: La teoría emergente que representa la visión de la gestión del cuidado considera al profesional de enfermería del primer nivel de atención como líder con conocimientos, empoderado y actualizado en la práctica del cuidado dirigida a la persona, familia y comunidad con actitud empática y asertiva.

Palabras clave: cuidado, gestión, primer nivel de atención

\section{Abstract}

Objective: To analyze Care Management Vision of Nursing professionals who work in the first level of attention of in Health System Micro-Networks located in Tacna. The scene took place in the province and city of Tacna. The participants were 07 nursing professionals with positions or experience in the Head of micro networks and health establishments. Material and methods: Qualitative study of grounded theory. The technique was an in-depth interview and a semi-structured interview guide as an instrument. The sample was obtained from theoretical data saturation sampling. Results: In the analysis of the information, the grounded theory method was used through the open, axial and selective coding process in which five categories, 13 subcategories and 28 units of analysis emerged. The first category was related to disciplinary concepts; the second, to the professional judgment of care management; as a third category, the competence of the nursing professional for care management was considered; the fourth category was related to the community; finally, the fifth category was about leadership. From them emerged the theory that represents the vision of care management. Conclusions: The emerging theory that represents the vision of care management considers the nursing professional of the first level of care as a leader with knowledge, empowered and updated in the practice of care directed to the person, family and community, with an empathetic and assertive attitude.

Keywords: care, management, first level of care

\footnotetext{
${ }^{1}$ Escuela Profesional de Enfermería, Universidad Nacional Jorge Basadre Grohmann

a Doctora en Ciencias: Enfermería
} 


\section{Resumo}

Objetivo: Analisar o significado da visão da gestão do cuidado dos profissionais de enfermagem que atuam no primeiro nível de atenção das micro redes de saúde de Tacna. A cena aconteceu na província e na cidade de Tacna. Os participantes foram 07 profissionais de enfermagem com cargos ou experiência na chefia de micro redes e estabelecimentos de saúde. Material e métodos: Estudo qualitativo da teoria fundamentada. A técnica foi uma entrevista em profundidade e um roteiro de entrevista semiestruturada como instrumento. A amostra foi obtida a partir de amostragem de saturação teórica de dados. Resultados: $\mathrm{Na}$ análise das informações utilizou-se o método da teoria fundamentada nos dados por meio do processo de codificação aberta, axial e seletiva em que emergiram cinco categorias, 13 subcategorias e 28 unidades de análise. A primeira categoria estava relacionada aos conceitos disciplinares; a segunda, ao julgamento profissional da gestão do cuidado; como terceira categoria, considerou-se a competência do profissional de enfermagem para o gerenciamento do cuidado; a quarta categoria estava relacionada à comunidade; finalmente, a quinta categoria era sobre liderança. Deles emergiu a teoria que representa a visão da gestão do cuidado. Conclusões: A teoria emergente que representa a visão da gestão do cuidado considera o profissional de enfermagem de primeiro nível de atenção como um líder com conhecimento, empoderado e atualizado na prática do cuidado dirigido à pessoa, família e comunidade com uma atitude empática e assertiva.

Palavras-chave: cuidado, gestão, primeiro nível de atenção

\section{Introducción}

El cuidado es la esencia de la enfermería y, para tal efecto, el profesional de enfermería requiere conocimientos, actitudes, y destrezas propias; a la vez que utiliza herramientas de la gerencia para prever, organizar, dirigir y controlar el cuidado con calidad.

La finalidad de la actividad de la gestión de los cuidados está centrada en la interacción entre la persona y el entorno. Dicho de otro modo, está dirigida a mantener y promover los contextos favorables para el cuidado de la persona, lo que compromete a la enfermera a involucrarse activamente en la organización de la profesión y al mismo tiempo al desarrollo de las instituciones para las cuales presta sus servicios. Estas acciones, se fundamentan como en el eje disciplinar, es decir, el cuidado del individuo, la familia y la comunidad.

Según la Organización Mundial de la Salud (OMS) y la Organización Panamericana de la Salud (OPS) 2015, el personal de enfermería conforma más del $60 \%$ de la fuerza de trabajo en salud y cubre el $80 \%$ de las necesidades de atención. En la actualidad la enfermera(o) tiene mayor presencia en los niveles de atención I y II con diversos tipos de contrato. ${ }^{1}$

Para comprender el funcionamiento de una determinada organización o grupo social, como en el caso de los profesionales de enfermería del equipo de atención primaria, debemos centrarnos en los comportamientos que los caracterizan. ${ }^{2}$ Por esta razón es importante conocer las concepciones que la disciplina de enfermería tiene en relación a la gestión de los cuidados y hacer visibles tales características que configuran la organización del trabajo en un primer nivel de atención y así enriquecer los fundamentos de la profesión en la actualidad.

La información se obtuvo de profesionales de enfermería con cargo de jefatura en enfermería y profesionales asistenciales con experiencia en el cargo de las microredes de la Región de Tacna. Siendo un tipo de investigación cualitativa con metodología de teoría fundamentada, lo que permitió tanto obtener una teoría emergente como elaborar aproximaciones conceptuales 
de la visión de la gestión del cuidado de los profesionales de enfermería.

Para la recolección de datos, la técnica fue la entrevista a profundidad y; como instrumento, una guía de entrevista semiestructurada. Las entrevistas fueron grabadas y transcritas.

Para el análisis de la información se utilizó el método de teoría fundamentada. En el proceso de codificación, se obtuvieron cinco categorías que explican los conceptos disciplinares; es decir, el juicio profesional de la gestión del cuidado, las competencias del profesional de enfermería, la relación con la comunidad y la visión de la gestión del cuidado, de las cuales emergieron trece subcategorías: 1) persona como ser integral, 2) cuidado como compromiso, 3) salud como bienestar biopsicosocial, 4) entorno como todo lo que rodea a la persona, 5) gestión del cuidado enfocada en un proceso administrativo, 6) gestión del cuidado enfocada en la salud, 7) conocimientos, 8) comunicación asertiva, 9) empatía, 10) coordinación, 11) trabajo comunitario interinstitucional, 12) liderazgo en actualización y 13) liderazgo en el cuidado.

\section{Material y métodos}

La presente investigación es un estudio cualitativo con metodología de teoría fundamentada (TF) que es inicialmente un acercamiento a la colección de datos y análisis de datos desarrollada por Barney Glaser y Anselm Strauss en los años sesenta. Tiene sus orígenes en la sociología, en particular en el interaccionismo simbólico como base teórica. Este último se enfoca en los procesos de interacción entre las personas que exploran conductas humanas y roles sociales. EI interaccionismo simbólico explica como la interacción se produce entre seres humanos que interpretan o definen las acciones de los demás en lugar de reaccionar a las mismas. ${ }^{3}$

El desarrollo de esta metodología permite construir una teoría la que se encuentra subyacente en los datos que se obtienen en el campo investigado, permitiendo dar significado a los fenómenos que se desarrollan en una disciplina específica.

Sustenta que las personas son actores que adoptan un papel activo al responder a situaciones problemáticas. En este contexto, se concibe la idea de que las personas actúan con una intención y que esta intención se define y se redefine por la interacción. A esto se suma una sensibilidad a la naturaleza evolutiva y al desarrollo permanente de los acontecimientos (procesos), a la constancia de la relación entre las condiciones (la estructura), a la acción (el proceso) y las consecuencias. ${ }^{4}$

De las nueve cabeceras de micro red de Tacna, la muestra de estudio estuvo conformada por siete de ellas, en las cuales se desempeña un profesional de enfermería con el cargo de jefatura del servicio de enfermería. El tamaño de la muestra se determinó con base a las necesidades de información; por ello, uno de los principios que guía este estudio es un muestreo por saturación de datos, es decir, hasta el punto en que ya no se obtiene nueva información y esta comienza a ser redundante. Para la recolección de datos la técnica fue la entrevista a profundidad y como instrumento una guía de entrevista semiestructurada la cual permitió que los participantes (el profesional de enfermería) expresen todo aquello que va relacionado con el concepto de la gestión de cuidado y las acciones que realizan en el día a día desde su experiencia. Dichas entrevistas fueron grabadas y transcritas, se asignó a cada participante un código. Por ejemplo E-01, hasta E-07. Se analizó la información según la metodología con una codificación abierta codificación axial y la codificación selectiva.

\section{Resultados}

En la investigación realizada bajo el enfoque cualitativo de TF, se analizaron los discursos de los participantes mediante el proceso de codificación abierta, axial y selectiva, con una metodología inductiva; dicho de otra forma, se interpretaron los significados de la realidad laboral del personal de enfermería del primer nivel de atención. 
Los procedimientos que proporcionan estandarización y rigor al desarrollo de la investigación son llamados procesos de codificación siendo la codificación abierta el proceso analítico por medio del cual se identifican los conceptos y se descubren en los datos sus propiedades y dimensiones. En este proceso se revisaron los datos recolectados (entrevistas, observaciones, anotaciones). Luego, se analizaron y se generaron categorías iniciales de significado a través de comparación constante. Se eliminó la redundancia y se desarrolló evidencias para las categorías. Se identificaron subcategorías, las cuales fueron codificadas. Luego se procedió a realizar la codificación axial que es el proceso de relacionar las categorías a sus subcategorías, denominado así porque la codificación ocurre alrededor del eje de una categoría, y enlaza las categorías en cuanto a sus propiedades y dimensiones. En este proceso las categorías se construyen de manera sistemática y se enlazan a las subcategorías. Para esto, se construyó un esquema analizando las concepciones que lo describen. Por último, se realizó la codificación selectiva la cual integra y refina las categorías, aquí se analizó el esquema revisando las unidades para establecer la teoría emergente. Así, se obtuvo el sentido de entendimiento.

A través de estos procesos, se logró identificar 28 unidades de significado. Estas unidades fueron agrupadas en 13 subcategorías, de las cuales emergieron 05 categorías que se aproximan y caracterizan la visión de la gestión del cuidado del profesional de enfermería del primer nivel de atención.

\section{Cuadro 1}

Identificación de categorías, subcategorías y unidades de significado

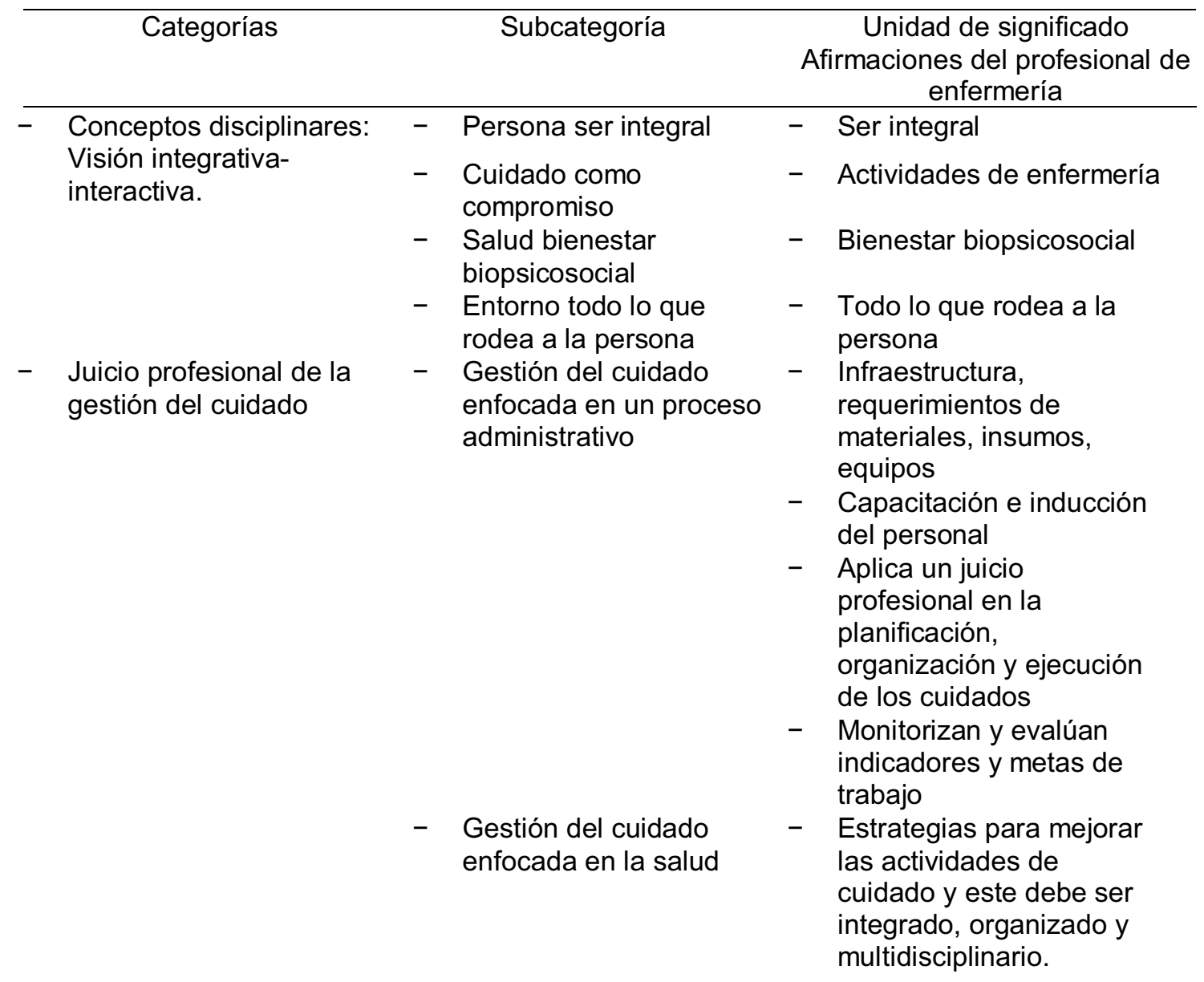




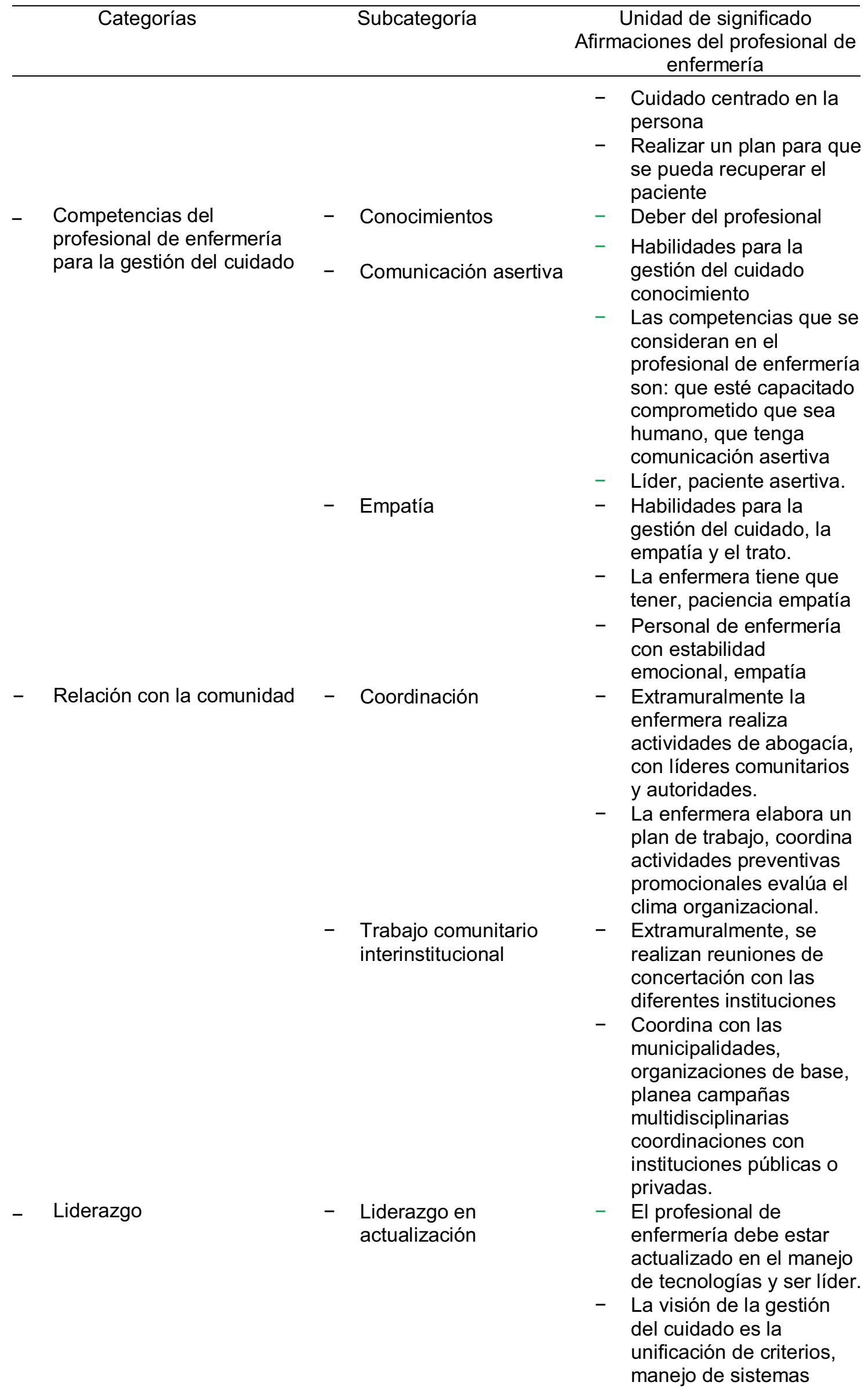




\begin{tabular}{|c|c|c|c|}
\hline Categorías & & Subcategoría & $\begin{array}{c}\text { Unidad de significado } \\
\text { Afirmaciones del profesional de } \\
\text { enfermería }\end{array}$ \\
\hline & & Liderazgo en el cuidado & $\begin{array}{l}\text { informáticos, armonía en } \\
\text { el trabajo, trabajo en } \\
\text { equipo, y mejores } \\
\text { relaciones } \\
\text { interpersonales. } \\
\text { - } \quad \text { Implementación de } \\
\text { sistemas de información } \\
\text { oportuna y veraz, mejorar } \\
\text { el tiempo de espera de } \\
\text { los pacientes, continuar } \\
\text { con los sistemas de pre } \\
\text { citas. } \\
\text { Grupo para compartir } \\
\text { experiencias talleres de } \\
\text { liderazgo, pasantías } \\
\text { Motivar el liderazgo, } \\
\text { empoderamiento en los } \\
\text { profesionales de } \\
\text { enfermería mediante } \\
\text { capacitaciones. } \\
\text { El profesional de } \\
\text { enfermería debe tener la } \\
\text { capacidad de liderar el } \\
\text { grupo. }\end{array}$ \\
\hline
\end{tabular}

\subsection{Construcción de categorías}

Según la metodología, el siguiente paso consiste en crear categorías. Por tanto, se detallarán cada una de las categorías a continuación.

\subsubsection{Conceptos disciplinarios: visión integrativa-interactiva}

Se debe mencionar que los metaparadigmas se encuentran enmarcados en grandes corrientes de pensamientos 0 maneras de ver o comprender el mundo, los cuales han sido llamados paradigmas. Estos paradigmas tienen su influencia sobre las concepciones de la disciplina de enfermería. Según Newman ${ }^{5}$ estos son: visión particular determinante o de reacción, visión integrativainteractiva y la visión unitaria- transformadora.

Esta categoría surge del significado que otorgan los enfermeros a los conceptos metaparadigmaticos, persona, cuidado, salud y entorno en el primer nivel de atención.
El metaparadigma es un componente abstracto de la jerarquía estructural del conocimiento de enfermería. Está constituido por los conceptos que identifican los fenómenos de interés de la enfermería, además se representan los conceptos 0 núcleos básicos.

\subsubsection{Juicio profesional de la gestión del cuidado}

Esta categoría surge de la concepción del profesional de enfermería sobre la gestión del cuidado que con sus propias palabras expresan las acciones que desempeñan en el primer nivel de atención.

Generalmente, se reconoce que el rol de una gestora consiste en planificar, organizar, dirigir y controlar los recursos financieros, humanos y materiales con la finalidad del logro de objetivos institucionales. ${ }^{6}$

Meleis y Jenring $^{6}$ sostienen que la gestión de los cuidados se ejerce no solamente en función a teorías administrativas prestadas 
de otras disciplinas, sino igualmente en relación a las concepciones propias de la disciplina de enfermería. Dichas concepciones sirven de base para su práctica.

Meleis ${ }^{6}$ sostiene que la dirección de los cuidados debe tener una visión clara y explícita de la disciplina enfermera con el fin de contribuir a la solución de problemas relativo a los cuidados, a los clientes y al personal.

\subsubsection{Competencias del profesional de enfermería para la gestión del cuidado}

El concepto de competencia surge de la necesidad de valorar no solo el conjunto de los conocimientos apropiados (saber) y las habilidades y destrezas (saber hacer) desarrollados por una persona, sino de apreciar su capacidad de emplearlas para responder a situaciones, resolver problemas y desenvolverse en el mundo.

Las competencias que desarrolla el profesional de enfermería no son conocimientos $\mathrm{o}$ habilidades fragmentadas, sino un conjunto de saberes combinados, que el mismo profesional construye a partir de la experiencia de la secuencia de actividades. Todas estas competencias que el desarrolla son de importancia para la adaptación a los cambios, generando dentro de la gestión del cuidado una vía para mejorar la calidad de atención de la enfermería. ${ }^{8}$

\subsubsection{Relación con la comunidad}

La estrategia de atención primaria de salud cobra un significado especial cuando se examina las relaciones entre la comunidad y las instituciones de salud. El cambio fundamental es que la comunidad y los individuos dejen de ser objeto de atención y se conviertan en actores, que conocen, participan y toman decisiones sobre su propia salud y asuman responsabilidades específicas ante ella.

Los factores ideológicos, políticos, sociales y culturales influirán en este proceso y condicionarán la claridad con la que se vean las relaciones entre la salud y otros aspectos de la vida de la comunidad. Es así que las actividades del sector salud se integran en un enfoque intersectorial en la medida que la comunidad participe de manera efectiva en todos los aspectos de su propio desarrollo y los demás sectores social y económico. ${ }^{9}$

\subsubsection{Liderazgo}

El liderazgo es la habilidad de influir en las personas de modo que trabajen con entusiasmo y con la perspectiva de alcanzar los objetivos que se cree son para el bien común, el líder es aquel que ejerce autoridad y liderazgo, es una persona honesta y confiable, buen modelo, cuidadoso, comprometido, buen oyente, mantienen la responsabilidad en las personas, las trata con respeto, las incentiva y presenta una actitud positiva y entusiasta y le agrada a la gente. ${ }^{10}$

\subsection{Construcción de la teoría emergente}

Luego del análisis de cada categoría surge la construcción teórica sobre la visión de la gestión del cuidado de los profesionales de enfermería en el primer nivel de atención de la región de Tacna según su manera de ver y comprender las concepciones de la disciplina de enfermería su visión es:

Ser un Profesional de Enfermería en el primer nivel de atención líder con conocimientos, empoderado y actualizado en la práctica del cuidado dirigida a la persona, familia $y$ comunidad con actitud empática $y$ asertiva.

\section{Discusión}

Para comprender el constructo de la teoría emergente es necesario discutir cada una de las subcategorías obtenidas de las narrativas de los profesionales de enfermería las cuales se encuentran esquematizadas según la estructura del cuidado de la Teoría de los cuidados de Kristen M. Swanson. ${ }^{11}$ 


\section{Figura 1}

\section{Estructura del cuidado de Kristen M. Swanson Adaptado para representar esquemáticamente la teoría emergente}

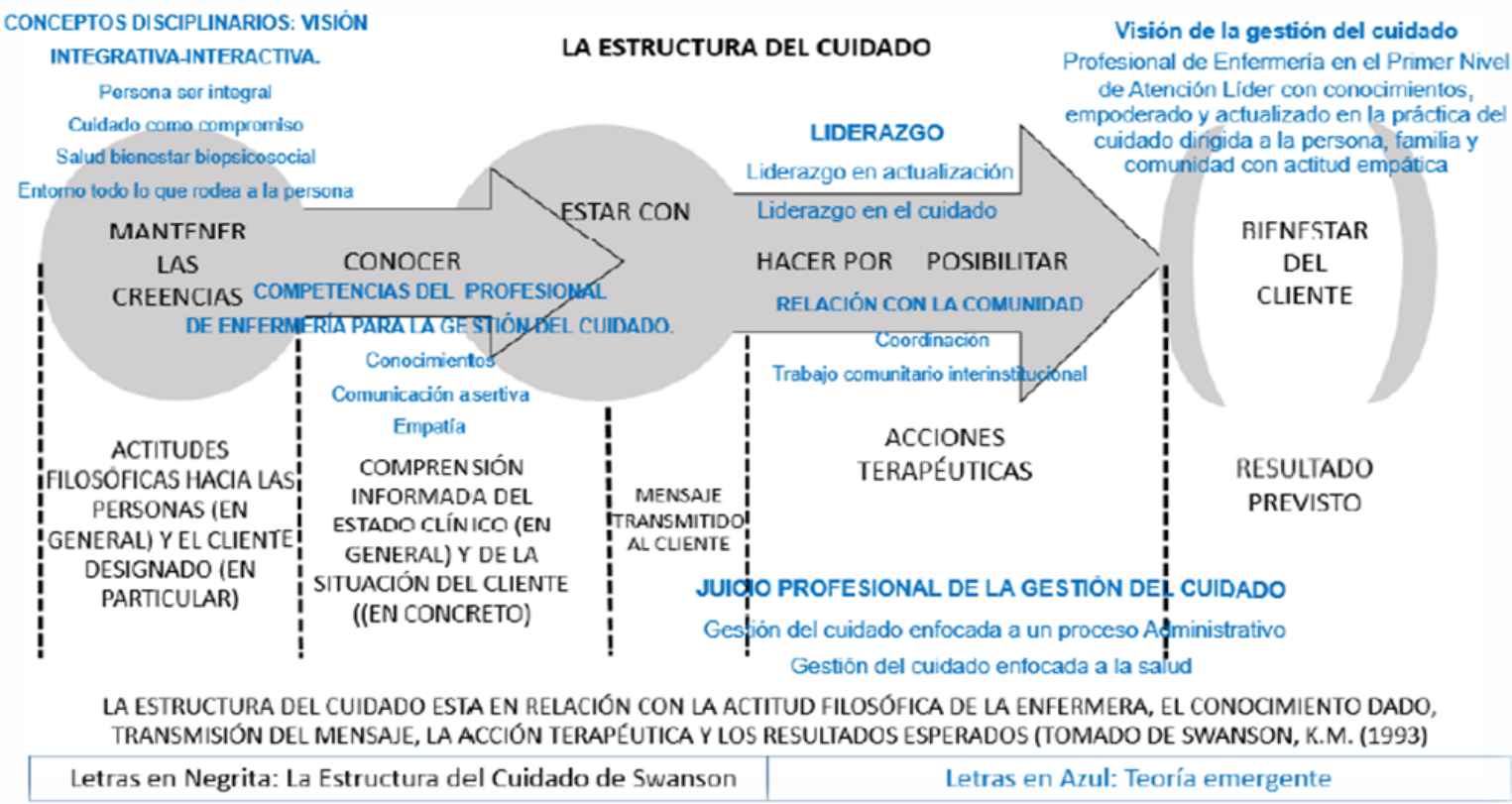

\subsection{Manifestar las creencias}

Se representa en el esquema los conceptos disciplinarios identificados como una visión Integrativa-Interactiva subcategorizado como:

\subsubsection{Persona ser integral}

Según los principales conceptos de Swanson, ${ }^{11}$ las personas son descritas como seres únicos que están en un proceso de creación y cuya integridad se completa cuando se manifiestan en pensamiento, sentimientos y conductas.

Narrativas representativas:

Persona es ese ser integral de nuestra sociedad por el cual debemos velar por su bienestar físico, psíquico, social (E-3 L2-3)

En cuanto a la persona es ver al mismo paciente como una persona, verlo como un todo integral (E-4 L7)

\subsubsection{Cuidado como compromiso}

Para Boff, ${ }^{12}$ el cuidar es más que un acto es una actitud. Es lo que se opone al desinterés y la indiferencia, abarca más que un momento de atención de celo y develo. Representa una actitud de ocupación, preocupación, de responsabilidad y compromiso afectivo con el otro.

Narrativas representativas:

Los cuidados que nosotros como personal de salud generamos en esta persona, en este ser en este paciente o este usuario externo que nosotros concebimos en este primer nivel de atención. (E-3 L12)

El cuidado son aquellas actividades que uno realiza con la finalidad de poder mejorar el estado actual de una persona, de una sociedad. (E-5 L4)

\subsubsection{Salud bienestar biopsicosocial}

Según la OMS la salud es un estado de completo bienestar físico, mental y social, y no 
solamente la ausencia de afecciones o enfermedades. La cita procede del preámbulo de la Constitución de la Organización Mundial de la Salud, que fue adoptada por la Conferencia Sanitaria Internacional en 1946 . $^{13}$

Boff $^{12}$ entiende la salud no como un estado sino como un proceso permanente de búsqueda de equilibrio dinámico de todos los factores que componen la vida humana, dichos factores están al servicio de la persona para que tenga la fuerza de ser persona autónoma, libre, abierta y creativa en las diferentes situaciones que tenga que enfrentar.

Narrativas representativas:

Salud está relacionado con el completo bienestar físico psíquico y social de una persona, aunque no podamos cumplir en un cien por ciento con esa definición. (E-3 L3-4)

La salud es el bienestar individual de una persona como también el aspecto social, en el aspecto psicológico, un equilibrio entre estos escenarios 0 factores. (E-5 L2-3)

\subsubsection{Entorno todo lo que rodea a la persona}

Según Swanson ${ }^{11}$ el entorno lo define de manera situacional, sostiene que para la enfermería es cualquier contexto que influye o que está influido por el cliente, existiendo muchos tipos de influencia sobre el entorno como los aspectos culturales, sociales, biofísicos, políticos económicos y otros.

Según los discursos el entorno está descrito como todo lo que rodea a la persona y a la familia, el lugar donde se desenvuelven identificado como un espacio físico.

Narrativas representativas:

El entorno son los escenarios o lo que rodea a la persona con lo cual la persona se interrelaciona, podría ser la familia, la comunidad, la iglesia las instituciones públicas o privadas ( $\mathrm{E}-5$ L5-7)

\subsection{La acción terapéutica}

Representa en el esquema el juicio profesional de la gestión subcategorizado como:

\subsubsection{Gestión del cuidado enfocada en un proceso administrativo}

A través de la administración, se busca el uso eficiente de los recursos para lograr un objetivo. En el caso de los servicios del primer nivel de atención de salud, el desafío es dirigir estos recursos a satisfacer las necesidades de una sociedad que se encuentra en constante evolución y cambio.

Este proceso administrativo se inicia con el estudio de las necesidades, la demanda, la oferta, el diagnostico. Es importante tener la información básica, procesarla y canalizarla para determinar tanto los principales problemas de salud como la capacidad operativa existente y la que se puede generar potencialmente. Con estos elementos, es posible determinar las prioridades de intervención para luego programar y ejecutar las actividades de cuidado. ${ }^{9}$

Narrativas representativas:

La gestión del cuidado es amplia porque enfermería no tan solo se desenvuelve en la atención directa al paciente, sino que también tenemos que ver que en cuanto a todo lo que involucra la atención, la infraestructura, los equipos, la capacitación que tenga el personal una serie de procesos que van a ir directamente relacionados al cuidado y la atención del paciente. (E-1 L 7-11)

\subsubsection{Gestión del cuidado enfocada en la salud}

Según Kerouac, ${ }^{6}$ la dirección del cuidado está llamada a promover los enfoques centrados en la salud más que en la enfermedad, lo que modificara la elección del 
sistema de prestaciones de cuidados, las calificaciones, y el número de personal cuidador, las descripciones del puesto de trabajo, las normas y los criterios de calidad de los cuidados, los programas de orientación y de formación continuada, el sistema de promoción y las afectaciones propuestas.

Narrativas representativas:

En cuanto a la gestión del cuidado es la base del quehacer de la enfermera, es realizar todo un plan donde se tenga que determinar todo lo que se tenga que hacer con el paciente para que se pueda recuperar definitivamente en todas sus esferas. (E-4 L 12-15)

\subsection{Conocer}

Representa en el esquema las competencias del profesional de Enfermería para la gestión del cuidado subcategorizada como:

\subsubsection{Conocimientos}

Swanson ${ }^{11}$ define el conocimiento como el esfuerzo por comprender el significado de un suceso en la vida del otro, evitando conjeturas, centrándose en la persona a la que se cuida buscando claves, valorando meticulosamente y buscando un proceso de compromiso entre el que cuida y el que es cuidado.

Narrativas representativas:

La enfermera tiene que tener conocimientos, tiene que tener habilidades, experiencia que va adoptando a lo largo de su tiempo de trabajo. (E-04 L 19-20)

\subsubsection{Comunicación asertiva}

Para Bofff ${ }^{12}$ en la concretización del cuidado encontramos el cuidado del otro que nos indica que no existe solo la red de relaciones sociales, también existen las personas concretas, los hombres y las mujeres. Referente a los seres humano, las personas son seres hablantes y a través del habla construyen el mundo con sus relaciones y el yo solo se constituye mediante el dialogo con el tú. El tú asiste al alumbramiento del yo.

El cuidado se da entonces velando porque este dialogar yo-tú sea liberador cinegético y constructor de una alianza perenne de paz y amor. ${ }^{12}$

La comunicación no es solo dar información, sino también una manera de relacionarse, en la cual el profesional de enfermería está presente con todo su ser, como testigo empático, con un compromiso de ayudar y acompañamiento al ser humano en su realidad. ${ }^{14}$

Es importante destacar que en el proceso de comunicación, la enfermera desarrolla ciertas conductas y aptitudes, algunas de ellas las mencionaremos a continuación:

El profesional de enfermería es capaz de iniciar conversaciones con el individuo, familiares y actores sociales de su comunidad siendo capaz de guiar la interacción desde un nivel superficial a uno más complejo, determina necesidades, comprende las percepciones, estimula a los participantes a expresar sus sentimientos en forma apropiada, considera la edad, el sexo, la cultura, las experiencias vitales, aptitudes y preferencias mientas realiza su plan de cuidados, escucha atentamente los mensajes, lucha por no estereotipar a los que la rodean, permite periodos de silencio en una conversación, alienta a discutir problemas a fondo, proporciona retroalimentación cuando sigue y comprende la conversación, interroga con habilidad y gentileza considerando la situación y sentimientos, busca la aclaración cuando es necesario, observa gestos, expresiones faciales, apariencia postura, tono de voz y fluidez de su interlocutor, presta atención a los contenidos que se repiten en una conversación, detecta incongruencias, nota efectos en la condición física, emocional social y espiritual de individuo y familia, estimula a ser activos en el proceso de cuidado de la salud, 
valora los mensajes y otros datos para determinar la necesidad de asistencia, establece metas, explora diversos enfoques para dar solución a la problemática, considera el factor de disposición favorable para comunicarse, respeta la confianza cuando recibe información confidencial, colabora con otros miembros del equipo para el logro de un nivel óptimo de salud, busca asistencia con otros miembros del equipo de salud, considera el ambiente familiar y de la comunidad donde habita el individuo, utiliza recursos para la asistencia, busca conocimientos y habilidades adicionales para desempeñarse competente y compasivamente. ${ }^{15}$

Esta lista de conductas y aptitudes demuestra la complejidad del proceso de comunicación, muestra también los numerosos y variados aspectos de las actividades diarias de una enfermera en la práctica de su profesión al realizar intervenciones a nivel individual, familiar y comunitario.

\section{Narrativas representativas:}

Después también tenemos personal que tenga comunicación asertiva entre ella y el paciente ese es otro aspecto que podemos determinar cómo enfermeras. (E-02 L 20-22)

La Enfermera debe ser líder, debe ser paciente, asertiva, comunicativa, tomar en cuenta las opiniones de los demás. (E-05 L 10-11)

\subsubsection{Empatía}

La práctica de enfermería es mucho más que el cumplimiento de metas. Requiere de la capacidad de ponerse en el lugar del otro. La empatía es definida como una respuesta emocional derivada de la percepción del estado o condición de otra persona, siendo congruente con esa situación. ${ }^{16}$

Boff ${ }^{17}$ hace mención en las repercusiones del cuidado de la compasión radical la que comprende como una contribución del budismo que no significa tener pena por el otro, la compasión tiene para el dos significados fundamentales el primero es respetar al otro, no invadir su espacio, dejarlo crecer y el segundo significado es si el otro sufre nunca dejarlo solo. Es entonces que en el reconocimiento del semejante comienza la empatía, comienza el cuidado como la relación profundamente humana que se prolonga hacia la naturaleza.

Swanson ${ }^{11}$ hace referencia en sus principales conceptos el Estar con que significa estar emocionalmente presente con el otro. Incluye estar allí en persona, transmitir disponibilidad y compartir sentimientos sin abrumar a la persona cuidada y el Hacer porque significa hacer por otros lo que se haría por uno mismo, si fuera posible, incluyendo adelantarse a as necesidades, confortar, actuar con habilidad y competencia y proteger al que es cuidado, respetando su dignidad.

Es entonces esa actitud necesaria que se advierte en los gestos y en las palabras del profesional de enfermería y que comunica al paciente que su situación y preocupaciones nos importan. Con la empatía mostramos que hemos entendido lo que siente la otra persona y que somos sensibles en cada momento al contenido emocional de lo que nos está comunicando. ${ }^{14}$

Narrativas representativas:

Lo otro es también la paciencia y sobre todo cualidades como la empatía, ponerse en el lugar de esa persona, verlo como si fuera algún hermano o un familiar. (E-04 L21-22)

\subsection{Posibilitar}

Representada en el esquema como la relación con la comunidad, subcategorizado como:

\subsubsection{Coordinación}

Durante el proceso en el cual el profesional de enfermería realiza la planificación, organización, ejecución y evaluación de actividades aplica estrategias de 
coordinación intra y extra sectoriales. Teniendo en cuenta que durante este proceso el equipo de salud debe de reflexionar sobre el objetivo institucional la adecuación de la organización estructural y funcional a la realidad del ámbito jurisdiccional.

El profesional de enfermería en la atención primaria además de brindar cuidados de salud debe actuar como un coordinador de un equipo de trabajo que tiene funciones de promoción y prevención de la salud, eso requiere de una actitud activa y de tomar la iniciativa.

\subsubsection{Trabajo comunitario interinstitucional}

Vemos que en todos los discursos, la enfermera identifica el trabajo comunitario como un trabajo en el que debe estar coordinando permanentemente con los actores e instituciones que forman parte de la jurisdicción en la que desempeña su labor.

Según el modelo de atención integral en salud basado en familia y comunidad en el eje de necesidades las intervenciones en la comunidad constituyen la oferta de actividades de promoción de la salud y prevención de la enfermedad, que están orientadas a atender las necesidades de salud de la comunidad en sus diferentes escenarios: instituciones educativas, centros laborales, redes sociales y otros. Esta oferta de intervenciones tiene la finalidad de lograr el desarrollo de comunidades con entornos saludables.

\footnotetext{
Narrativas representativas

Creación de alianzas con todos los sectores, organizaciones de base, vasos de leche club de madres, pronois Instituciones educativas iniciales, privadas o estatales, municipios, nosotros actualmente trabajamos con municipios saludables, universidades privas o sea con todas las instituciones que nos rodean. (E-03 L 25-29)
}

\subsection{Hacer por}

Representa en el esquema el liderazgo, subcategorizado como:

\subsubsection{Liderazgo en actualización}

El profesional de enfermería reconoce que es importante contar con herramientas de comunicación que le permitan un mejor desarrollo y empoderamiento, relacionado a sistematizar la gran cantidad de datos que genera y este manejo sea más efectivo a través de un liderazgo en el manejo de la tecnología de la comunicación.

\begin{abstract}
Narrativas representativas:
La visión de la gestión del cuidado es contar con Profesionales capacitados, actualizados con las últimas tecnologías con actitud positiva, no tan solo tener el conocimiento, la habilidad la destreza en la atención integral del paciente de su familia de la comunidad y del entorno nosotros manejamos todo, todo lo que está alrededor del paciente integral. (E-01 L43-47)
\end{abstract}

\subsubsection{Liderazgo en el cuidado}

Según Kerouac ${ }^{6}$ indica que la gestión de los cuidados requiere la utilización del potencial de cada persona cuidadora para dispensar lo más eficazmente posible los cuidados dirigidos a la salud de los diversos clientes y por extensión, los de la comunidad, siendo el rol de la enfermera gestora el de liderazgo y de motivación procesos esenciales para la gestión del entorno social.

\footnotetext{
Narrativas representativas:

Nosotros siempre buscando el liderazgo ser pues en algún momento líder en el cuidado en el primer nivel de atención que es preventivo promocional. (E-03 L 39-40)
} 
Al análisis de toda la información analizada a través de la metodología de Teoría Fundamentada tenemos la siguiente teoría emergente: Un profesional de enfermería en el primer nivel de atención líder con conocimientos, empoderado y actualizado en la práctica del cuidado dirigida a la persona, familia y comunidad con actitud empática y asertiva.

\section{Referencias}

1. Organización Mundial De La Salud. Organización Panamericana de La Salud. La OPS/OMS. Destaca la necesidad de formar más personal de enfermería en América Latina y el Caribe. [Internet].2015 [citado Septiembre 2018 ]. shorturl.at/BIO56

2. Bengoa R, Nuño R. Curar y cuidar. Innovación en la gestión de enfermedades crónicas: Una guía práctica para avanzar. Barcelona: Elservier Masson; 2008. 162 p. ISBN-13: 9788445823125

3. Organización Panamericana de la Salud. Investigación cualitativa en enfermería. Contexto y bases Conceptuales. Serie Paltex. Salud y Sociedad 2000 Numero 9; 2008

4. Strauss A, Corbin J. Bases de la investigación cualitativa. Técnicas y procedimientos para desarrollar la teoría fundamentada. Colombia. Editorial Universidad de Antioquia. Facultad de Enfermería de la Universidad de Antioquia; 2002

5. Grupo Cuidado. El arte y la ciencia del cuidado. Colombia Editora Guadalupe Ltda: 2002

6. Kérouac S. Pepin J. Ducharme F. EI pensamiento enfermero. Barcelona. Editorial Masson, S.A; 1995

7. Ministerio para Educación Nacional de Colombia. Competencias laborales: Base para mejorar la empleabilidad de las personas; 1993.
8. Gonzales C. Competencias profesionales en enfermeras que desempeñan su labor en una unidad de cuidados intensivos. Rev. iberoam. Educ. investi. Enferm. 2015; 5(1):35-46.

9. Kroeger A. Luna R. Atención primaria de salud. Principios y Métodos. 2da ed. México: Editorial Pax . Organización Panamericana de la salud; 1992.

10. Waldow V. Cuidar: expresión humanizadora. 1ra ed. México.: Editorial palabra ediciones; 2008.

11. Raile M. Modelos y Teorías en Enfermería. 8va ed. España: Editorial: Elsevier; 2015.

12. BOFF L. El cuidado esencial. Ética de lo humano compasión por la tierra. Colección Estructura y Procesos. Serie Religión. España. Ed. Trotta.;2002

13. Organización Mundial de La Salud. Preguntas más frecuentes. ¿Cómo define la OMS la Salud? 14. [Internet].2018 [citado septiembre 2018]; Disponible en: https://www.who.int/es/about/who-weare/frequently-asked-questions

14. Valverde G. Comunicación terapéutica en enfermería. 1ra ed. España: Editorial. Difusión avance en enfermería; 2007

15. Maurren J. Comunicación y relaciones en enfermería. Editorial El manual Moderno S.A. DE C.V. Méxic; 1983.

16. Marques A. Koller S. Competência social e empatia: um estudo sobre resiliência com crianças em situação de pobreza. 1 Universidade Federal do Rio Grande do Sul. Estudos de Psicologia Competência Social e Empatia, 5(1), 71-93

17. Boff L. Toro B. Foro de Emprendedurismo Social en la nueva economía. Panel Empatía y Cuidado: Paradigmas de la nueva civilización. Forte de Copacabana, Río de Janeiro. 2012 [Citado: 2018 Julio ]; Disponible en: https://www.youtube.com/watch?v=rBSD5 Yiof6o

\section{Correspondencia:}

ecachicatariv@unjbg.edu.pe
Fecha de recepción: 12 de agosto de 2020

Fecha de aceptación: 19 de noviembre 2020 